\title{
Evidence for a metal-poor population in the inner Galactic bulge
}

\author{
M. Schultheis ${ }^{1}$, K. Cunha ${ }^{2}$, G. Zasowski ${ }^{3,4}$, A. E. García Pérez ${ }^{5}$, K. Sellgren ${ }^{6}$, V. Smith ${ }^{7,2}$, D. A. García-Hernández ${ }^{5,8}$, \\ O. Zamora ${ }^{5,8}$, T. K. Fritz ${ }^{9}$, F. Anders ${ }^{10,11}$, C. Allende Prieto ${ }^{5,8}$, D. Bizyaev ${ }^{12,13}$, K. Kinemuchi ${ }^{12}$, K. Pan ${ }^{12}$, \\ E. Malanushenko ${ }^{12}$, V. Malanushenko ${ }^{12}$, and M. D. Shetrone ${ }^{14}$
}

1 Laboratoire Lagrange, Université Côte d'Azur, Observatoire de la Côte d'Azur, CNRS, Bvd de l'Observatoire, 06304 Nice, France e-mail: mathias.schultheis@oca.eu

2 Observat'orio Nacional, 77 Sao Cristóvao, 20921-400 Rio de Janeiro, Brazil

3 Department of Physics \& Astronomy, Johns Hopkins University, Baltimore, MD 21218, USA

4 NSF Astronomy \& Astrophysics Postdoctoral Fellow

5 Instituto de Astrofísica de Canarias, Calle Vía Láctea s/n, 38205 La Laguna, Tenerife, Spain

${ }^{6}$ Department of Astronomy, The Ohio State University, 140 West 18th Avenue, Columbus, OH 43210, USA

7 National Optical Astronomy Observatories, Tucson, AZ 85719, USA

8 Departamento de AstrofÃnysica, Universidad de La Laguna (ULL), 38206 La Laguna, Tenerife, Spain

9 Department of Astronomy, University of Virginia, Charlottesville, 3530 McCormick Road, VA 22904-4325, USA

10 Leibniz-Institut für Astrophysik Potsdam (AIP), An der Sternwarte 16, 14482 Potsdam, Germany

${ }^{11}$ Laboratǿrio Interinstitucional de e-Astronomia, - LIneA, Rua Gal. Joseé Cristino 77, 20921-400 Rio de Janeiro, Brazil

12 Apache Point Observatory and New Mexico State University, PO Box 59, Sunspot, NM, 88349-0059, USA

13 Sternberg Astronomical Institute, Moscow State University, 119992 Moscow, Russia

14 University of Texas at Austin, 32 Fowlkes Rd, McDonald Observatory, Tx 79734-3005, USA

Received 21 July 2015 / Accepted 16 October 2015

\section{ABSTRACT}

\begin{abstract}
The inner Galactic bulge has, until recently, been avoided in chemical evolution studies because of extreme extinction and stellar crowding. Large, near-IR spectroscopic surveys, such as the Apache Point Observatory Galactic Evolution Experiment (APOGEE), for the first time allow the measurement of metallicities in the inner region of our Galaxy. We study metallicities of $33 \mathrm{~K} / \mathrm{M}$ giants situated in the Galactic center region from observations obtained with the APOGEE survey. We selected K/M giants with reliable stellar parameters from the APOGEE/ASPCAP pipeline. Distances, interstellar extinction values, and radial velocities were checked to confirm that these stars are indeed situated in the inner Galactic bulge. We find a metal-rich population centered at $[\mathrm{M} / \mathrm{H}]=+0.4 \mathrm{dex}$, in agreement with earlier studies of other bulge regions, but we also discovered a peak at low metallicity around $[\mathrm{M} / \mathrm{H}]=-1.0$ dex. This finding suggests the presence of a metal-poor population, which has not previously been detected in the central region. Our results indicate a dominant metal-rich population with a metal-poor component that is enhanced in the $\alpha$-elements. This metal-poor population may be associated with the classical bulge and a fast formation scenario.
\end{abstract}

Key words. Galaxy: bulge - stars: fundamental parameters - stars: abundances - Galaxy: stellar content

\section{Introduction}

The Milky Way bulge is such a complex system that its formation and evolution are still poorly understood. As a result of high extinction and crowding, the study of the Galactic bulge remains challenging. Extinction of more than $30 \mathrm{mag}$ in $A_{\mathrm{V}}$ in the Galactic center (GC) regions requires IR spectroscopy. While more and more detailed chemical abundances in the intermediate and outer bulge (such as Baade's Window) are now available thanks to the large spectroscopic surveys ARGOS (Freeman et al. 2013), Gaia-ESO (Rojas-Arriagada et al. 2014), and APOGEE (García Pérez et al. 2013), chemical abundances of stars in the inner Galactic bulge (IGB) with projected distances of $R_{\mathrm{G}} \leq 200 \mathrm{pc}$ from the $\mathrm{GC}$ remain poorly studied.

Most of the chemical abundance studies in the IGB have been limited to luminous supergiants whose complex and extended stellar atmospheres make abundance analysis difficult. Carr et al. (2000), Ramírez et al. (2000), Cunha et al. (2007), and Davies et al. (2009) analyzed high-resolution spectra of supergiant stars in the GC region and found metallicities of near solar metallicity. A similar conclusion was obtained by Najarro et al. (2009) after analyzing two luminous blue variables. Cunha et al. (2007) derived abundances of nine giant and supergiant stars in the central cluster and found a metallicity of $[\mathrm{Fe} / \mathrm{H}]=+0.14 \pm 0.06$, together with enhanced $[\mathrm{O} / \mathrm{Fe}]$ and $[\mathrm{Ca} / \mathrm{Fe}]$ abundances. The total range in $\mathrm{Fe}$ abundance among GC stars, 0.16 dex, is significantly narrower than the iron abundance distributions found in the literature for the older bulge population. Ryde \& Schultheis (2015, RS2015) studied nine field stars with a projected distance $R \leq 50 \mathrm{pc}$. They found a metal-rich population with $[\mathrm{Fe} / \mathrm{H}]=+0.11 \pm 0.15$ and low $\alpha$-value, and a lack of a metal-poor population, similar to Cunha et al. (2007). Their mean metallicities are $\sim 0.3$ dex higher than fields in the inner bulge (Rich et al. 2007, 2012), indicating that the GC region contains a distinct population. Grieco et al. (2015) compared these data with a chemical evolution model and concluded that in order to reproduce the observed $[\alpha / \mathrm{Fe}]$ ratios, the GC region should have experienced a main strong burst of star formation and should have evolved very quickly with an IMF which contains more massive stars. All these studies comprise a 


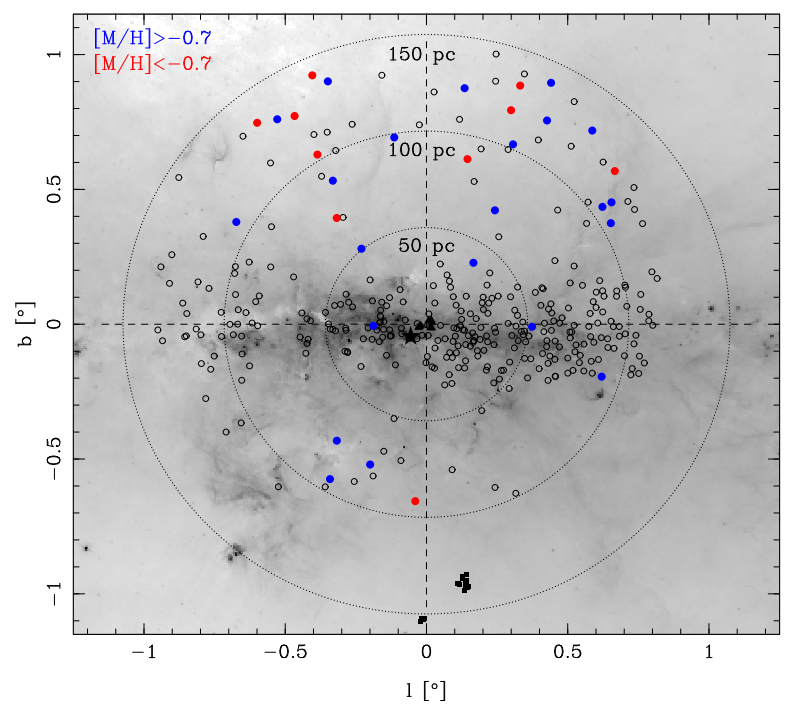

Fig. 1. Galactic longitude vs. Galactic latitude distribution of the GALCEN sample superimposed on the $8 \mu \mathrm{m}$ IRAC image of GLIMPSE-II (Churchwell et al. 2009). The red filled symbols indicate our selected sample with $[\mathrm{M} / \mathrm{H}]<-0.7$, the blue filled symbols with $[\mathrm{M} / \mathrm{H}]>-0.7$, and the open black symbols the full GALCEN sample. Circles denote galactocentric radii of 50,100 , and $150 \mathrm{pc}$, assuming $R_{\odot}=8 \mathrm{kpc}$. The black asterisk show the GC field of Cunha et al. (2007), the black triangles those of Ryde \& Schultheis (2015), and the black squares those of Rich et al. (2007).

limited number of targets with precise chemical abundances. In this paper, we discuss the metallicity distribution of 33 APOGEE $\mathrm{K} / \mathrm{M}$ giants in the so-called GALCEN field, increasing the statistics of $\mathrm{K} / \mathrm{M}$ giant stars with reliable metallicities significantly.

\section{The sample}

\subsection{APOGEE}

The Apache Point Observatory Galactic Evolution Experiment (APOGEE; Majewski et al. 2015), one of four Sloan Digital Sky Survey-III (SDSS-III, Eisenstein et al. 2011) experiments, is a large scale, near-IR, high-resolution $(R \sim 22500)$ spectroscopic survey of Milky Way stellar populations. The survey uses a dedicated, 300-fiber, cryogenic spectrograph that is coupled to the wide-field, Sloan $2.5 \mathrm{~m}$ telescope (Gunn et al. 2006) at the Apache Point Observatory (APO). APOGEE observes in the $H$-band, where extinction by dust is significantly lower than at optical wavelengths (e.g., $A(H) / A(V) \sim 0.16)$.

With its high resolution and high S/N $(\sim 100$ per Nyquistsampled pixel), APOGEE determined both accurate radial velocities (better than $0.5 \mathrm{~km} \mathrm{~s}^{-1}$ ) and reliable abundance measurements, including the most abundant metals in the universe $(\mathrm{C}, \mathrm{N}$, $\mathrm{O})$, along with other $\alpha$, odd- $Z$, and iron-peak elements. The latest SDSS-III data release (DR12; Ahn et al. 2014) provides to the scientific community spectra of more than 140000 stars, as well as the derived stellar properties, including radial velocities, effective temperatures, surface gravities, metallicities, and individual abundances. Additional information, such as photometry and target selection criteria, is also provided and described in Zasowski et al. (2013).

\subsection{The GALCEN field}

The GALCEN APOGEE field is centered at $(l, b)=\left(0.173^{\circ}\right.$, $-0.07^{\circ}$ ) with a field of view of $\sim 2.9$ sq. deg (see Fig. 1), and it

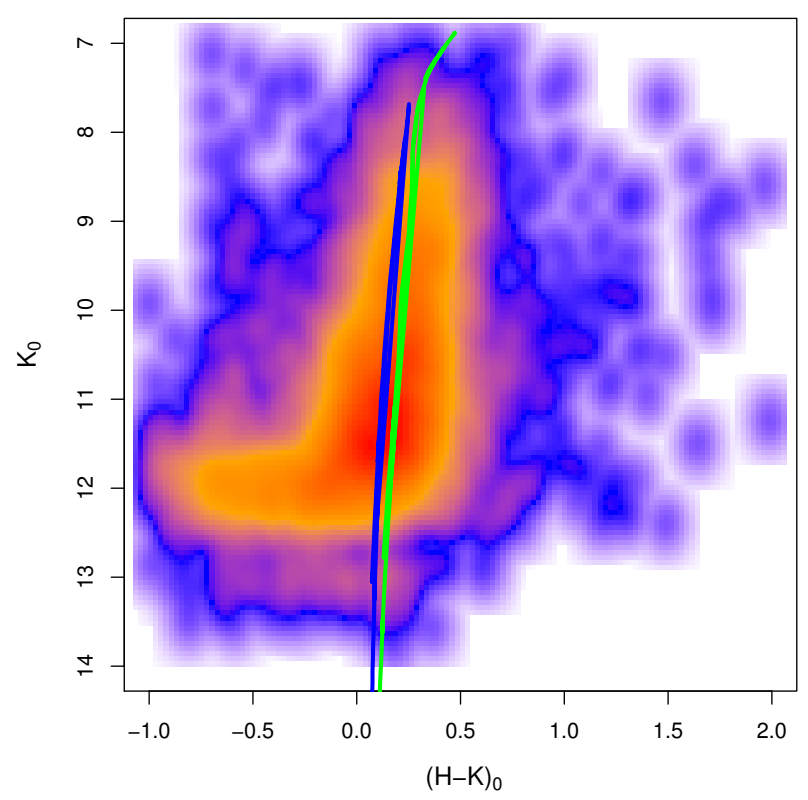

Fig. 2. Dereddened $(H-K)_{0}$ vs. $K_{0} 2 \mathrm{MASS}$ diagram using the extinction map of Schultheis et al. (2014) centered at $l=0^{\circ}$ and $b=0.65^{\circ}$. The blue line shows the Padova isochrones of $10 \mathrm{Gyr}$ with $[\mathrm{Fe} / \mathrm{H}]=-0.4$, while the green line $[\mathrm{Fe} / \mathrm{H}]=+0.3$. Black circles show our sample of stars dereddened with the RJCE method. Open circles show $[\mathrm{Fe} / \mathrm{H}]>-0.7$, while the filled circles are the low-metallicity stars with $[\mathrm{Fe} / \mathrm{H}]<-0.7$.

consists of 339 stars. Owing to the high interstellar extinction (see, e.g., Schultheis et al. 2014), a special target selection procedure has been applied for the GALCEN field (Zasowski et al. 2013). Forty-eight stars are known to be long-period variables based on $K$-band lightcurves (Matsunaga et al. 2009). Thirtyfour stars are asymptotic giant branch (AGB) stars based on lowresolution $H K$-band spectra (Schultheis et al. 2003). In addition, spectroscopically known supergiants such as IRS7, IRS19, and IRS22 were observed as well as supergiant candidates based on photometric criteria. These AGB and supergiant targets have among the coolest effective temperatures $\left(T_{\text {eff }}<3500 \mathrm{~K}\right)$ in the APOGEE stellar sample. Figure 1 shows the distribution of the APOGEE GALCEN sample in Galactic coordinates. We only used $\sim 10 \%$ of the 339 GALCEN stars (filled circles) for our analysis (Sect. 3).

Figure 2 shows the dereddened color-magnitude diagram $\left(\left(H-K_{0}\right)\right.$ vs. $\left.K_{0}\right)$ of the selected GALCEN sources (black points), together with the 2MASS data in this field that were dereddened using the high-resolution VVV extinction map of Schultheis et al. (2014). The GALCEN sample sources on the other hand, were dereddened using the RJCE method on a star-by-star basis (see Zasowski et al. 2013 for more details). Superimposed are the Padova isochrones with an age of $10 \mathrm{Gyr}$ and $[\mathrm{Fe} / \mathrm{H}]=-0.4$ and $[\mathrm{Fe} / \mathrm{H}]=+0.3$.

\subsection{Stellar parameters}

Stellar parameters and chemical abundances of up to 15 elements are determined by the ASPCAP pipeline. These values are based on a $\chi^{2}$ minimization between observed and synthetic model spectra (see Zamora et al. 2015; Holtzman et al. 2015 for more details on the DR12 spectral libraries) performed with the FERRE code (Allende Prieto et al. 2006, and subsequent updates). Model spectra are interpolated on a regular grid computed with the 
ASS $\epsilon$ T code (Koesterke 2009) for a custom line list especially compiled for the survey (Shetrone et al. 2015), and a large grid of model atmospheres (Mészáros et al. 2012).

The accuracies of the ASPCAP $T_{\text {eff }}, \log g$, and $[\mathrm{M} / \mathrm{H}]$ values were evaluated by Holtzman et al. (2015) with a sample of wellstudied field and cluster stars, including a large number of stars with asteroseismic stellar parameters from NASA's Kepler mission (Borucki et al. 2010). In this work, we use DR12 calibrated stellar parameters (we refer to Holtzman et al. 2015 for details).

\section{Sample selection}

In order to ensure the most reliable stellar parameters and abundances, we selected stars in the GALCEN field from the DR12 dataset with following criteria: (i) ASPCAP $\chi^{2}<20$; (ii) PARAMFLAG $=0$ for $T_{\text {eff }}, \log g$, and $[\mathrm{M} / \mathrm{H}]$, meaning that we avoid stars that are too close to the edge of the model grid; and (iii) $\log g<3$ to avoid foreground dwarfs. We exclude known supergiants, AGBs or possible AGB/supergiant candidates (based on near-and mid-IR criteria) for our analysis but restrict ourselves to $\mathrm{K} / \mathrm{M}$ giants. In total we are left with $33 \mathrm{~K} / \mathrm{M}$ giants. We checked the reliabilty of the ASPCAP fits to the observed spectra for each star by visual inspection. Because of the cool effective temperatures of our stars (see Fig. 3), we restricted ourselves to using temperatures, surface gravities, metallicities, and global $\alpha / \mathrm{Fe}$ abundances, rather than individual elemental abundances. We added an additional cut in effective temperature $\left(T_{\text {eff }}>3700 \mathrm{~K}\right)$ for the analysis of the $\alpha$-elements to avoid possible systematic abundance effects in cool stars.

Figure 3 shows the distribution of the sample stars in $T_{\text {eff }}$ vs. $\log g$ space, colored by metallicity. One sees clearly that our sample covers a wide metallicity range and that the majority of our targets are $\mathrm{M}$ giants. As we exclude cooler stars with $T_{\text {eff }}<3600 \mathrm{~K}$, we could be missing more metal-rich stars. We calculated spectrophotometric distances with the Bayesian approach of Anders et al. (2014) to be sure that our stars are really located in the IGB. Our stars are indeed located at a heliocentric distance of $8 \mathrm{kpc}$ with a typical uncertainty on the order of $20 \%$. No difference in the distance distribution was found between metal-rich and metal-poor stars. In addition, our derived extinction values are compatible within $20 \%$ with those of the IGB. We also studied the radial velocity distribution of our sample, which has a mean value of $-32 \mathrm{~km} \mathrm{~s}^{-1}$ with a dispersion of $122 \mathrm{~km} \mathrm{~s}^{-1}$. These values are similar to the velocity dispersion of $114 \mathrm{~km} \mathrm{~s}^{-1}$ obtained by Zhu et al. (2008) for the central parsec of the Galaxy and to the results obtained in the study of Rich et al. (2007), with a velocity dispersion of $115 \pm 15 \mathrm{~km} \mathrm{~s}^{-1}$. This is in perfect agreement with the predicted dispersion by the $N$-body bulge model of Shen et al. (2010). Interestingly, there is one metal-poor $[\mathrm{Fe} / \mathrm{H}]=-1.0$ high radial velocity star with $v_{\text {rad }}=356 \mathrm{~km} \mathrm{~s}^{-1}$. Proper motions would be necessary to derive the orbital period of this star.

\section{Results and discussion}

Figure 4 shows the histogram of the GALCEN sample metallicities. For comparison we show the sample of Rich et al. (2007) in green and that of Ryde \& Schultheis (2015) in blue. We also show the metallicity distribution from the central cluster sample of Cunha et al. (2007) in red, although this should be considered as a different stellar population. We clearly see a prominent peak in the metallicity distribution at $\sim 0.4 \mathrm{dex}$, which

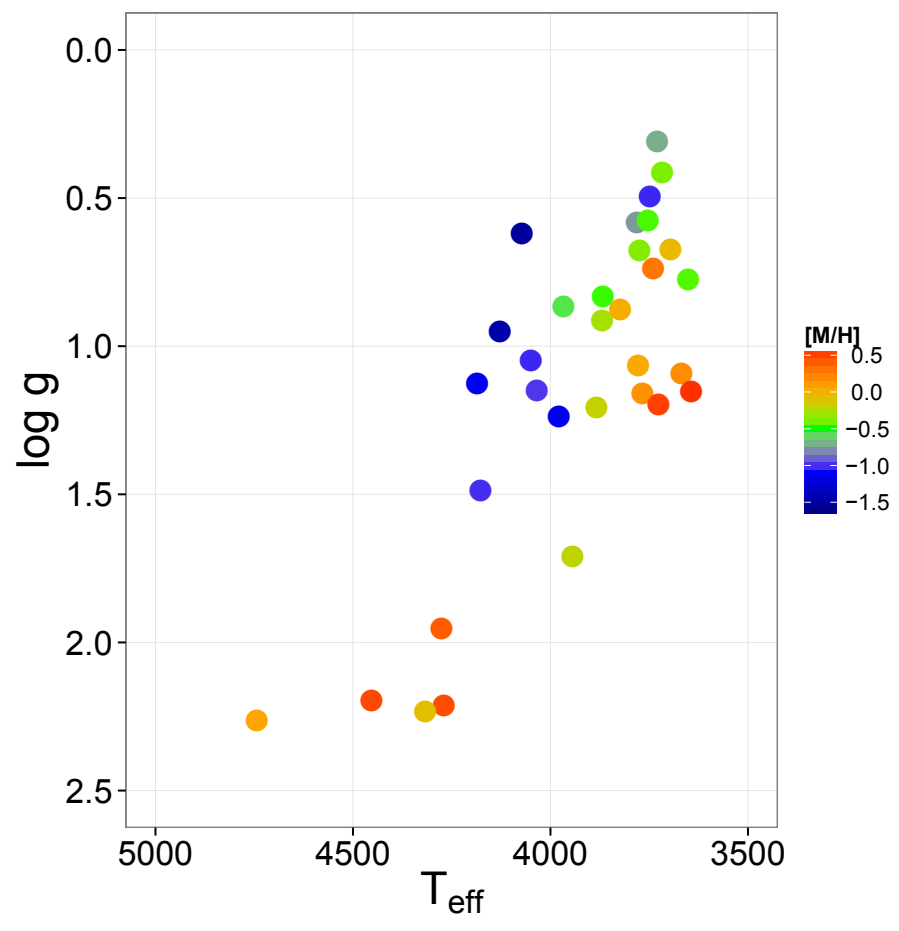

Fig. 3. Teff vs. $\log g$ as function of $[\mathrm{M} / \mathrm{H}]$ for our selected sample.

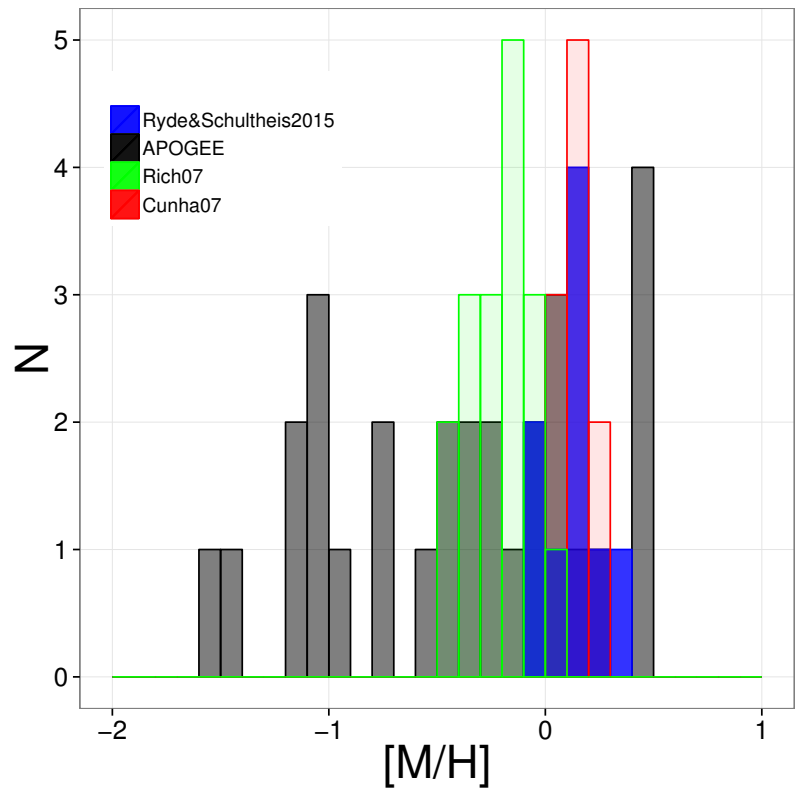

Fig. 4. Histogram of the metallicities compared to Rich et al. (2007) in green, Cunha et al. (2007) in red and Ryde \& Schultheis (2015) in blue. The bin size is 0.1 dex.

is about $0.2-0.3$ dex more metal rich than found in Ryde \& Schultheis (2015) and Rich et al. (2007). The most striking feature in the metallicity distribution is the large dispersion, with a rms scatter of 0.55 dex, which is much larger than seen in previous studies $(\sim 0.15 \mathrm{dex})$, and the presence of a metal-poor peak at $[\mathrm{Fe} / \mathrm{H}] \sim-1.0$ which has been observed by Babusiaux et al. (2014). This metal-poor population could be indicative of a classical bulge and associated with a fast formation scenario (Babusiaux et al. 2010). A manual analysis of the ${ }^{12} \mathrm{C} /{ }^{13} \mathrm{C}$ ratio in one of the metal-poor stars (2M17480557-2918376) reveals a value of ${ }^{12} \mathrm{C} /{ }^{13} \mathrm{C}=5 \pm 2$. This low carbon isotope ratio 


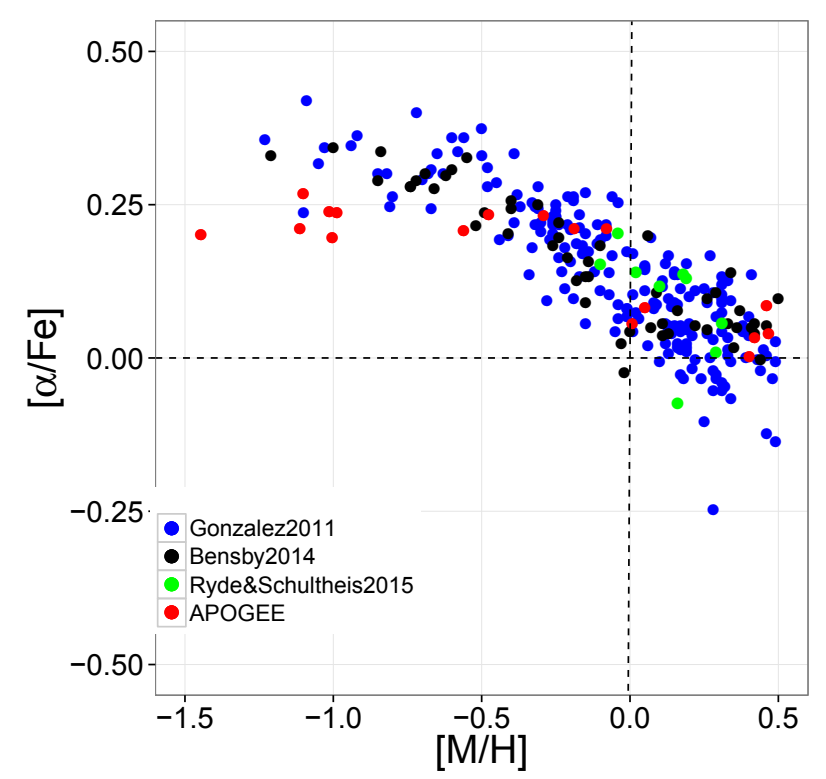

Fig. 5. $\alpha$ vs. $[\mathrm{M} / \mathrm{H}]$. The blue filled circles indicate the Galactic bulge data from Gonzalez et al. (2011), black from Bensby et al. (2013), green from Ryde \& Schultheis (2015), and in red those of our GALCEN sources. We used only stars with $T_{\text {eff }}>3700 \mathrm{~K}$.

for a red giant star is indicative of a low-mass first ascent giant $\left(M \sim 0.8-1.0 M_{\odot}\right)$ as first quantified by Gilroy (1989) for open cluster giants and confirmed by Mikolaitis et al. (2012). This value is close to that found in typical globular cluster red giants (e.g., Briley et al. 1994) which typify an old, low-mass population. Contrary to Ryde \& Schultheis (2015), Rich et al. (2007), and Cunha et al. (2007), the metallicity distribution resembles a typical bulge population such as that seen by Bensby et al. (2013), Hill et al. (2011), etc. for bulge fields at higher latitudes $\left(b>4^{\circ}\right)$. The metal-rich peak of our studied sample agrees with the metal-rich peak of the Galactic bulge sample of Bensby et al. (2013) and the red clump bulge sample of Hill et al. (2011). However, the second peak in their metallicity distribution is at $\sim-0.5$ to $-0.6 \mathrm{dex}$ (compared to $[\mathrm{M} / \mathrm{H}] \sim-1.0$ in our sample) with a lack of metal poor stars with $[\mathrm{M} / \mathrm{H}]<-1$. Our derived metallicity dispersion is comparable to that of Bensby et al. (2013; 0.53 dex) but larger than the red clump distribution of Hill et al. (2011) in Baade's Window.

Why has this metal-poor population not been revealed by other studies? The Ryde \& Schultheis (2015) and Rich et al. (2007) work contain a much smaller sample, which could be one reason that they have just missed these metal-poor stars. They deredden their targets using global extinction maps such as Schultheis et al. (1999), Schultheis et al. (2009), or Gonzalez et al. (2012). On the contrary, APOGEE calculates the reddening values on a star-by-star basis using a combination of near and mid-IR photometry and applies a homogenous $(J-K)_{0}>0.5$ color cut (see Zasowski et al. 2013 for further details).

Figure 5 shows the $\alpha$-abundances of our sample stars as a function of $[\mathrm{M} / \mathrm{H}]$. As a comparison we also show the Galactic bulge sample of the dwarfs and subgiants stars of Bensby et al. (2013), the M giants sample in the GC of Ryde \& Schultheis (2015), and the red clump star sample in Baade's Window of Gonzalez et al. (2011). Our $\alpha$-abundances agree with those of Ryde \& Schultheis (2015), Gonzalez et al. (2011) and Bensby et al. (2013) on the metal-rich side. However, the $\alpha$-elements of Bensby et al. (2013) and Gonzalez et al. (2011) are systematically higher than our $\alpha$-elements for low-metallicity stars $([\mathrm{Fe} / \mathrm{H}]<-0.5)$.

None of the current models is able to reproduce the observed metal-poor peak seen in our metallicity distribution. Grieco et al. (2015) calculated for the first time a chemical evolution model for the inner $200 \mathrm{pc}$ and compared it with the dataset of Ryde $\&$ Schultheis (2015). In order to produce their observed $[\alpha / \mathrm{Fe}]$ ratios and their metallicity distribution, they required a model assuming a main early strong burst of star formation with a star formation efficiency of $25 \mathrm{Gyr}^{-1}$ and a heavy IMF. However, their MDF consists of a single metal-rich population.

Regarding the explanation for other metal-poor bulge peaks/populations, Grieco et al. (2012), for example, used a chemical evolution model with two distinct populations that reproduces the MDF of Hill et al. (2011) in Baade's window. They concluded that the metal-poor population (which is different than the one discovered here) was formed on a very short timescale associated with an intense burst of star formation and a high star formation efficiency, while the metal-rich population was formed on a longer timescale and is related to the Galactic bar. Tsujimoto \& Bekki (2012) modeled the MDF with a twocomponent disk model: a metal-poor component with a very short timescale of $1 \mathrm{Gyr}$ and identical to the solar-neighborhood thick disc, and a metal-rich component with a longer timescale and a top-heavy IMF.

This shows that chemical evolution models can reproduce observed MDFs but fail to predict MDFs in different parts of our Galaxy. Thus, detailed confrontation with chemical evolution models are necessary to understand the origin of the metal-poor peak.

Acknowledgements. Funding for SDSS-III has been provided by the Alfred P. Sloan Foundation, the Participating Institutions, the National Science Foundation, and the US Department of Energy Office of Science. The SDSS-III web site is http://www.sdss3.org/. SDSS-III is managed by the Astrophysical Research Consortium for the Participating Institutions of the SDSS-III Collaboration including the University of Arizona, the Brazilian Participation Group, Brookhaven National Laboratory, Carnegie Mellon University, University of Florida, the French Participation Group, the German Participation Group, Harvard University, the Instituto de Astrofisica de Canarias, the Michigan State/Notre Dame/JINA Participation Group, Johns Hopkins University, Lawrence Berkeley National Laboratory, Max Planck Institute for Astrophysics, Max Planck Institute for Extraterrestrial Physics, New Mexico State University, New York University, Ohio State University, Pennsylvania State University, University of Portsmouth, Princeton University, the Spanish Participation Group, University of Tokyo, University of Utah, Vanderbilt University, University of Virginia, University of Washington, and Yale University.

\section{References}

Ahn, C. P., Alexandroff, R., Allende Prieto, C., et al. 2014, ApJS, 211, 17 Allende Prieto, C., Beers, T. C., Wilhelm, R., et al. 2006, ApJ, 636, 804 Anders, F., Chiappini, C., Santiago, B. X., et al. 2014, A\&A, 564, A115 Babusiaux, C., Gómez, A., Hill, V., et al. 2010, A\&A, 519, A77 Babusiaux, C., Katz, D., Hill, V., et al. 2014, A\&A, 563, A15 Bensby, T., Yee, J. C., Feltzing, S., et al. 2013, A\&A, 549, A147 Borucki, W. J., Koch, D., Basri, G., et al. 2010, Science, 327, 977 Briley, M. M., Smith, V. V., \& Lambert, D. L. 1994, ApJ, 424, L119 Carr, J. S., Sellgren, K., \& Balachandran, S. C. 2000, ApJ, 530, 307 Churchwell, E., Babler, B. L., Meade, M. R., et al. 2009, PASP, 121, 213 Cunha, K., Sellgren, K., Smith, V. V., et al. 2007, ApJ, 669, 1011 Davies, B., Origlia, L., Kudritzki, R.-P., et al. 2009, ApJ, 694, 46 Eisenstein, D. J., Weinberg, D. H., Agol, E., et al. 2011, AJ, 142, 72 Freeman, K., Ness, M., Wylie-de-Boer, E., et al. 2013, MNRAS, 428, 3660 García Pérez, A. E., Cunha, K., Shetrone, M., et al. 2013, ApJ, 767, L9 Gilroy, K. K. 1989, ApJ, 347, 835

Gonzalez, O. A., Rejkuba, M., Zoccali, M., Valenti, E., \& Minniti, D. 2011, A\&A, 534, A3

Gonzalez, O. A., Rejkuba, M., Zoccali, M., et al. 2012, A\&A, 543, A13

Grieco, V., Matteucci, F., Pipino, A., \& Cescutti, G. 2012, A\&A, 548, A60 
M. Schultheis et al.: Evidence for a metal-poor population in the inner Galactic bulge

Grieco, V., Matteucci, F., Ryde, N., Schultheis, M., \& Uttenthaler, S. 2015, MNRAS, 450, 2094

Gunn, J. E., Siegmund, W. A., Mannery, E. J., et al. 2006, AJ, 131, 2332

Hill, V., Lecureur, A., Gómez, A., et al. 2011, A\&A, 534, A80

Holtzman, J. A., Shetrone, M., Johnson, J. A., et al. 2015, AJ, 150, 148

Koesterke, L. 2009, in AIP Conf. Ser. 1171, eds. I. Hubeny, J. M. Stone, K. MacGregor, \& K. Werner, 73

Majewski, S. R., Schiavon, R. P., Frinchaboy, P. M., et al. 2015, arXiv e-prints [arXiv: 1509.05420]

Matsunaga, N., Kawadu, T., Nishiyama, S., et al. 2009, MNRAS, 399, 1709

Mészáros, S., Allende Prieto, C., Edvardsson, B., et al. 2012, AJ, 144, 120

Mikolaitis, S., Tautvaišienè, G., Gratton, R., Bragaglia, A., \& Carretta, E. 2012, A\&A, 541, A137

Najarro, F., Figer, D. F., Hillier, D. J., Geballe, T. R., \& Kudritzki, R. P. 2009, ApJ, 691, 1816

Ramírez, S. V., Sellgren, K., Carr, J. S., et al. 2000, ApJ, 537, 205

Rich, R. M., Origlia, L., \& Valenti, E. 2007, ApJ, 665, L119
Rich, R. M., Origlia, L., \& Valenti, E. 2012, ApJ, 746, 59

Rojas-Arriagada, A., Recio-Blanco, A., Hill, V., et al. 2014, A\&A, 569, A103

Ryde, N., \& Schultheis, M. 2015, A\&A, 573, A14

Schultheis, M., Ganesh, S., Simon, G., et al. 1999, A\&A, 349, L69

Schultheis, M., Lançon, A., Omont, A., Schuller, F., \& Ojha, D. K. 2003, A\&A, 405, 531

Schultheis, M., Sellgren, K., Ramírez, S., et al. 2009, A\&A, 495, 157

Schultheis, M., Chen, B. Q., Jiang, B. W., et al. 2014, A\&A, 566, A120

Shen, J., Rich, R. M., Kormendy, J., et al. 2010, ApJ, 720, L72

Shetrone, M., Bizyaev, D., Lawler, J., et al. 2015, ApJS, submitted [arXiv: 1502.04080 ]

Tsujimoto, T., \& Bekki, K. 2012, ApJ, 747, 125

Zamora, O., García-Hernández, D. A., Allende Prieto, C., et al. 2015, AJ, 149, 181

Zasowski, G., Johnson, J. A., Frinchaboy, P. M., et al. 2013, AJ, 146, 81

Zhu, Q., Kudritzki, R. P., Figer, D. F., Najarro, F., \& Merritt, D. 2008, ApJ, 681, 1254 